\title{
The use of Automated Mobile Sensors for Economical Intelligent Healthcare Systems with Body Area Network Assistance
}

\author{
Quience Gulati \\ M.Tech CS\&E \\ Amity University \\ Noida, India
}

\author{
Prachi Bhandari \\ M.Tech CS\&E \\ Amity University \\ Noida, India
}

\author{
Piyush Saxena \\ M.Tech CS\&E \\ Amity University \\ Noida, India
}

\begin{abstract}
Mobile Sensor Networks are constituted on ad-hoc basis by tiny mobile sensor nodes deployed randomly over an area. The automatic random localization of mobile nodes may serve many potential applications where dynamicity and continuity are critically important. In this paper we envision one such application of health management. In most of the severe health risk, the patient is kept under intensive observation to collect post treatment health data about the patient. Sometime the observation period is prolonged, and just contributes to the traffic in the hospital. Post treatment under the observation period a patient - equipped with various body sensors - may be shifted to a nearby affordable locality where a patient may perform unguided longer walk also. The body sensors will continuously be disseminating the various health data to a data repository in the hospital where the concerned doctor has the access to look it up.
\end{abstract}

As the sensor networks are formed on ad-hoc basis, so they are prone for loss. In our solution, we propose a random movement of a set of mobile sensor nodes between the body sensors and the repository and responsible to strengthen the ad-hoc wireless sensor network, the transmitting medium between them.

\section{Keywords}

Wireless Sensor Network, Health, Application

\section{INTRODUCTION}

Mobility models are important building blocks in simulationbased studies of wireless networks. This mobile sensor mobility model is a simple and straightforward model that describes the movement behavior of a mobile sensor node in a given system area. Each sensor node moves from an arbitrary location to a randomly selected destination with a randomly chosen speed in the range [vmin , ..., vmax]. Once the destination is reached, the node selects another destination and then continues to move with a different speed. Each node moves independent of its peers and the location to move next is chosen independent of its own previous movements. The time instance at which the node reaches the next coordinate points depends upon the previous time instance at the immediate previous coordinate, distance between the immediate previous coordinates and the current coordinates, and the velocity randomly chosen from the given limits. The measurement of the coordinates at any time instance depends upon a parameter also. In our paper we have termed it as $f$. The value of this parameter depends upon the tree time factors: the initial time of any movement, the ending time of any movement and the time instance of the interest. In our paper, it is assumed that the movements of the dynamic sensors are Brownian movements. The rest of the paper is organized as follows:

Section II, is the motivation section which focuses on the sensor mobility. This section covers- the random way point movement of sensor nodes in one or two dimension system space and a diagram, depicting the movement of the sensor node, describes the fact that the mobility of the sensors node improves the coverage in larger networks, Dynamic Coverage Maintenance scheme is touched. DCM compensates the coverage loss due to limited battery power of the sensor node, the advantages of mobile sensors on static sensors are discussed with the example, the three tiers architecture of a network in which few nodes are dynamic and few nodes are static, has been discussed in detail to get the impact of both the static and dynamic sensors in the same network, and discusses the application of mobile sensors in a very unique way. It discusses how the region (contour), where the pollutant concentration measurement is of great interest, is calculated. In section III, the simulation procedure of our work has been discussed in detail. Section IV, depicts the results of the simulations. Section V, concludes the paper.

\section{MOTIVATION}

In this paper a good number of papers are reviewed, that support the sensor mobility in the wireless sensor network. In most of the applications the dynamic sensors are more beneficial than the static sensor.

Random way point movement of the sensor node can be one or two dimension system space [1]. In one dimension a line segment is considered whereas in two dimension system a rectangular area of size $\mathrm{X} \times \mathrm{Y}$ or a circular area of radius $\mathrm{X}$ is considered.

The dynamic aspect of sensor node, depending on the sensor movement is an efficient approach in order to have better coverage of larger network area in between living facility and hospital [2]. As time goes by, a position is more likely to be covered; the targeted signals that are never be detected in a stationary sensor network can now be detected by moving sensors. The area coverage at specific time instants and during time intervals as well as the time it takes to detect a randomly located stationary target is characterized. This paper involves mobile targets, in our paper we have restricted up to static targets only as our main goal is to identify the position of sensor nodes at any time instants for further plan of actions. 


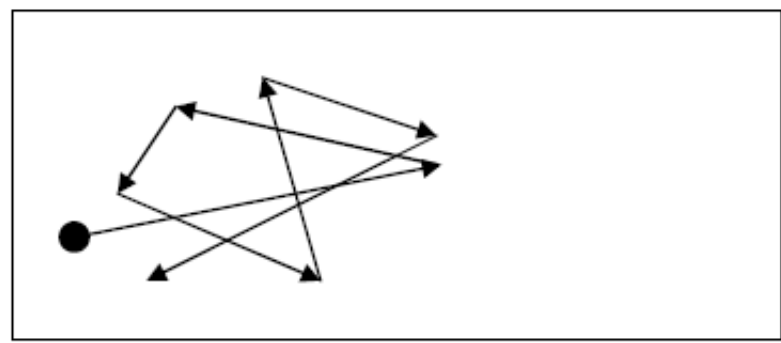

Fig. 1. Illustration of random movement of sensor node.

In Wireless Sensor Networks the coverage provided by sensor nodes is very crucial in order to enhance the monitoring of the health environment [3]. Many of the important health applications based on sensor networks demand autonomous mobility for the sensor nodes in order to strengthen the signals traffic and avoid any data loss. This paper [3] proposes the Dynamic Coverage Maintenance (DCM) schemes that exploit the limited mobility of the sensor nodes. The main objective of coverage maintenance is to compensate the loss of coverage with minimum expenditure of energy.

In traditionally sensor network, static sensor nodes are deployed to carry out the sensing operations [4]. If the monitored region is relatively large compared to the sensing range of a node, a large number of nodes are required in the region to achieve high coverage. The mobile nodes are the better alternative for this. The focus of this paper is on sensor networks in which the nodes are mobile. There are many applications for which sensor networks with mobile devices are better suited than a stationary network. The authors support this with an example. For instance, consider the problem of detecting a biochemical attack in a battlefield environment. The conventional stationary sensor network approach is to deploy a large number of devices with appropriate sensing capabilities in the battlefield. The nodes in the network sample their environment, exchange the sensed information, and fuse the information gathered to arrive at a consensus decision about the biochemical attack. If the battlefield area is large relative to either the sensing or the communication range of each device, the number of devices required to cover the entire region is also typically large. If the area of battlefield region is $12, r$ is the communication range of each device, and devices are uniformly and randomly deployed in the field, then $O\left(\frac{l^{2} \log l}{r^{2}}\right)$ devices will be needed to ensure network connectivity with sufficiently high probability [5]. An alternative, and possibly more attractive, approach is to deploy a network with mobile devices for dealing with the above problem. For instance, the troops and/or vehicles in the battlefield can carry the biochemical sensors while doing their normal daily activities. Since the troops and the vehicles are likely to be moving as part of their daily activities, the associated sensing devices are also on the move. These mobile sensing devices can sample the environment at potentially several different locations, exchange the information with other devices and/or fusion centre, and collaboratively make a decision for the presence of the biochemical attack.

Ameen et al. [6] explore the social issues involved using wireless sensor network for healthcare applications. In their work they mention that a human can be tracked and monitored using wearable and non-wearable sensor devices that gives rise to the potential issues of privacy and security. They further advocate the evolution and benefits of wireless sensor network infused in healthcare applications.
In their market dynamics report [7] halter et al. compare the monitory benefits vs. the today's investment on healthcare wireless sensor network, especially body area network. In their report they claim that by 2012 , the solutions offered by wireless sensor network for healthcare support could save \$25 billion annually by reducing the hospitalizations ad extending independent living during observation period for patient monitoring.

In [8], all the nodes in the network are not mobile but there are few mobile nodes present in the sensor field work as the forwarding agent. As a mobile node moves in close proximity to sensors, data is transferred to the mobile node for later depositing at the destination. This approach involves forming an ad-hoc network among the sensor nodes to send data. However, this faces the following energy related issues.

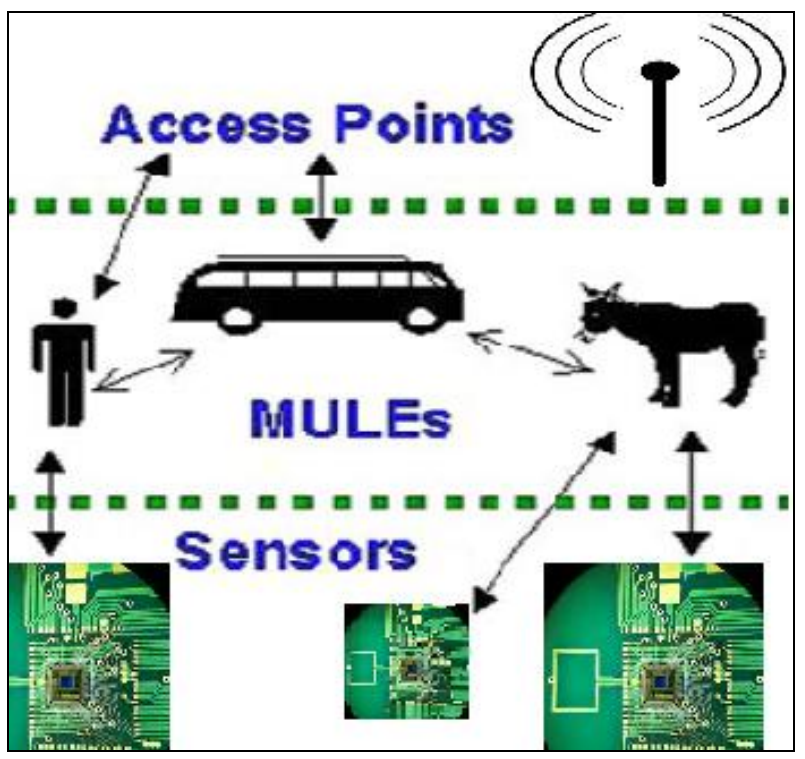

Fig. 2. The three tiers architecture of the network

Firstly, in a sparse network, the energy required for transmitting data over one hop is quite large. This is because sensors may be far from each other and the transmission power required increases as the fourth power of distance.

Secondly, in an ad-hoc network sensors have to not only send their data, but also forward data for other sensors.

Thirdly, the network has routing hotspots near the access points. Sensors that are near the access points have to forward many more packets and drain their battery much more quickly. Though In our paper, we do not consider the energy issue but this is the major problem to be considered in wireless sensor networks.

We assume the mobile entities consisting of the sensor nodes that receive the data from the body sensors and transmit tha to either next available node or to the destination centre hospital.

Mobile wireless sensor networks are helpful to examine the pollutant spill by tracing a contour of a particular concentration within a bounded region of varying pollutant concentration in a lake [9]. Tracking contours also helps in determining the rapidity of flow of contaminants and these results in providing an early warning for sensitive areas located in the vicinity of the spill. One way to architect a wireless sensor network to accomplish this task is to mount sensors on permanent moorings (static network) in the region where the spill has occurred and have each sensor measure the concentration of the pollutant at the location where it is 
mounted. An energy efficient algorithm would appropriate nodes in the network to estimate the contour with measurements made at the chosen nodes. On the other hand, one can also make use of mobile sensors, e.g. sensors mounted over rover buoys to move, sample and measure at different locations in the region. The use of mobile sensors is more efficient than that of static sensors in following means: (1) Improves sampling resolution - areas in the region which are unreachable for a static sensor network. (2) Eases deployment - in the region where measurements are taken and the sensors "intelligently" move and sample the region.(3) Increases adaptability to spatio-temporal dynamics of the phenomenon- Redeploying a static sensor network to do to be prohibitively expensive.

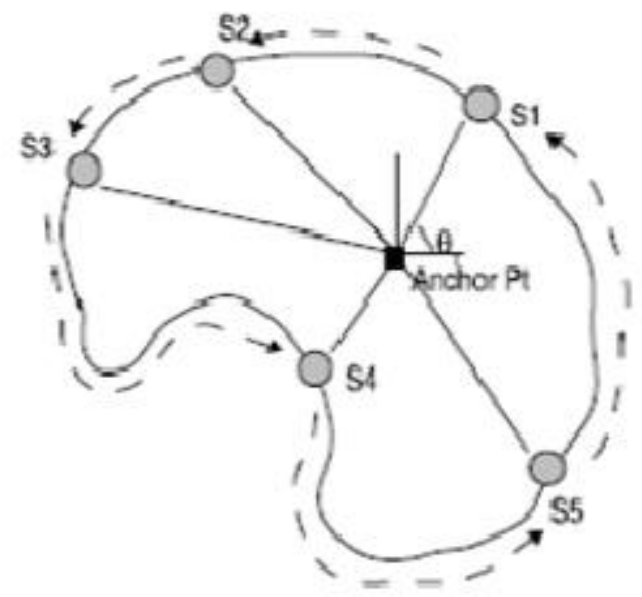

Fig. 3. The Movement of sensor nodes and the division of region in different sections centered at an Anchor $\mathbf{P t}$.

In this paper the centroid of the contour is taken as the anchor point. Once the sensors are deployed as shown in Figure 2 they communicate their initial location information to each other and they embed themselves in a ring i.e., one of the sensors, say, $\mathrm{S}_{1}$ is chosen as a lea d sensor and the one closest to it $\left(\mathrm{S}_{2}\right)$ is chosen as its anti-clock wise neighbor. Further, $\mathrm{S}_{2}$ picks the sensor $\left(\mathrm{S}_{1}\right.$ not inclusive) closest to it $\left(\mathrm{S}_{3}\right)$ and assigns it as its anti-clockwise neighbor and so on. The entire region $\mathrm{R}$ is divided into $\mathrm{N}$ sections cantered at a known anchor point. If $\theta_{1}$ is the current angle with respect to the centroid as the origin for sensor $S_{1}$, then the target angle of the $i^{\text {th }}$ sensor $\mathrm{Si}$ in the ring is given by $\theta_{1}+2 \pi(\mathrm{i}-1) / \mathrm{N}$.

If $\left(x_{c}, y_{c}\right)$ represents the location of the centroid, then the current angle of the $\mathrm{i}^{\text {th }}$ sensor at position, $\left(\mathrm{x}_{\mathrm{i}}, \mathrm{y}_{\mathrm{i}}\right)$ is computed as

$$
\theta_{i}\left(x_{i}, y_{i}\right)=\tan ^{-1}\left(\frac{y_{i}-y_{c}}{x_{i}-x_{c}}\right)
$$

The target angle for each sensor is its angle of approach towards the contour.

So this paper is one of the greatest motivations in the field of mobile sensors.

\section{SIMULATION PROCEDURE}

We consider a two dimensional sensor network field of size $\mathrm{X}_{\max } \times \mathrm{Y}_{\max }$. We further assume that there are $\mathrm{N}$ mobile sensor nodes in the network and total simulation time is SIMTIME.

Initially, the sensor nodes are distributed randomly by generating a sequence of random $\mathrm{x}$ and $\mathrm{y}$ co-ordinates, each in the range 0 to $X_{\max }$ and 0 to $Y_{\max }$. The set $\left(x^{1}{ }_{i}, y_{i}^{1}\right)$ be the initial co-ordinates, the location 1 of node $i$ at time $t^{1}$. For each node $i$, the value of $t$ is $0 \mathrm{sec}$ as it is the instant at which the simulation begins. Sensor node $i^{\text {th }}$ chooses a random destination location $\left(\mathrm{x}_{\mathrm{i}}^{1}, \mathrm{y}_{\mathrm{i}}^{1}\right)$ and a velocity $\mathrm{v}$ uniformly distributed in the range $\left[\mathrm{v}_{\min }, \ldots, \mathrm{v}_{\max }\right]$. The node then moves from $\left(\mathrm{x}_{\mathrm{i}}^{1}, \mathrm{y}_{\mathrm{i}}^{1}\right)$ to $\left(\mathrm{x}_{\mathrm{i}}^{2}, \mathrm{y}^{2}\right)$ with velocity $\mathrm{v}^{1-2}$.

The distance covered by mobile sensor node i during this movement is given by the following expression.

$d_{i}^{1-2}=\sqrt{\left(x_{1}^{i}-x_{2}^{i}\right)^{2}+\left(y_{1}^{i}-y_{2}^{i}\right)^{2}}$

The time instance at which the node $\mathrm{i}$ will reach the next coordinate $(x, y)$ is given by the following expression.

$t_{i}^{2}=t_{i}^{1}+\frac{d_{i}^{1-2}}{v_{i}^{1-2}}$

Once the mobile sensor node reaches the coordinate $\left(\mathrm{x}_{\mathrm{i}}^{2}, \mathrm{y}_{\mathrm{i}}^{2}\right)$, it chooses a random destination location $\left(\mathrm{x}_{\mathrm{i}}^{3}, \mathrm{y}_{\mathrm{i}}^{3}\right)$ and a velocity $\mathrm{v}^{2-3}$ i uniformly distributed over the range [vmin , $\ldots, \mathrm{vmax}]$. Now the node has acquired the sufficient parameter to make a move and it moves from $\left(\mathrm{x}_{\mathrm{i}}^{2}, \mathrm{y}_{\mathrm{i}}^{2}\right)$ to $\left(\mathrm{x}_{\mathrm{i}}^{3}, \mathrm{y}_{\mathrm{i}}^{3}\right)$ with that velocity $\mathrm{v}^{2-3}$.

The time instant at which node $\mathrm{i}$ will reach the coordinate point $(\mathrm{x}, \mathrm{y})$ is given by the following expression.

$t_{i}^{3}=t_{i}^{2}+\frac{d_{i}^{2-3}}{v_{i}^{2-3}}$

Thus ith node moves to sth location and the time instance is calculated as

$t_{i}^{S}=t_{i}^{S-1}+\frac{d_{i}^{(3-1)-3}}{v_{i}^{(2-1)-2}}$

where $\mathrm{t}_{i}{ }^{S-1}<$ SIM-TIME and $\mathrm{t}_{i}{ }^{S}>=$ SIM-TIME .

We have further defined a parameter named as fraction and represented by $\mathrm{f}$. This parameter has been used to calculate the coordinates between any given time instances.

For any $i^{\text {th }}$ node, to calculate the $\mathrm{X}$ and $\mathrm{Y}$ co-ordinates at a time instance $t$ between two time instances $t_{i}^{j}$ and $t^{k}{ }_{i}$ we define the fractional parameter $\mathrm{f}$ as

$f=\frac{t-t_{i}^{j}}{t_{i}^{k}-t_{i}^{j}}$

If $\left(\mathrm{x}_{\mathrm{i}}^{\mathrm{j}}, \mathrm{y}_{\mathrm{i}}^{\mathrm{j}}\right)$ and $\left(\mathrm{x}_{\mathrm{i}}^{\mathrm{k}}, \mathrm{y}_{\mathrm{i}}^{\mathrm{k}}\right)$ are the coordinates corresponding to the time instances $\mathrm{t}_{\mathrm{i}}^{\mathrm{j}}$ and $\mathrm{t}_{\mathrm{i}}^{\mathrm{k}}$ respectively.

Then at any $\mathrm{t}^{\text {th }}$ time instance the co-ordinates of for the $i^{\text {th }}$ node is calculated as follows

$x_{i}^{t}=f * x_{i}^{k}+(1-f) * x_{i}^{j}$

$y_{i}^{t}=f * y_{i}^{k}+(1-f) * y_{i}^{j}$ 


\section{SIMULATION RESULTS}

The results show the rectangular area $\mathrm{X} \times \mathrm{Y}$. In the following figures $4,5,6,7$ and 8 , the $\mathrm{X}$ axis is represented by $\mathrm{X}$ coordinate whereas $\mathrm{Y}$-axis is represented by $\mathrm{Y}$-coordinate. The dots inside the region are the mobile senor nodes. We have collected the snapshots at various time instances: 250 [figure4], 1250 [figure 5], 2250 [figure 6], 3250 [figure 7] and 4250 [figure 8]. At every instance we analyze that the organization of the sensor nodes in the region is changing at every time instance. Which means that the position of the sensors node is changing? If every node has a number then the changing position of that node can easily be identified.

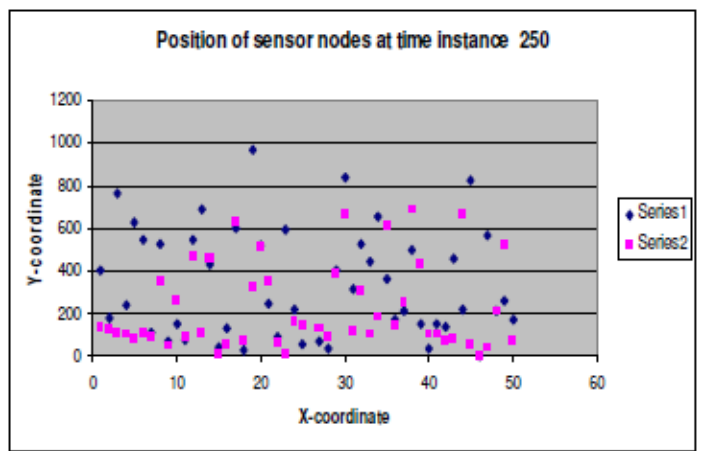

Fig. 4. Position of sensor nodes at time instance 250

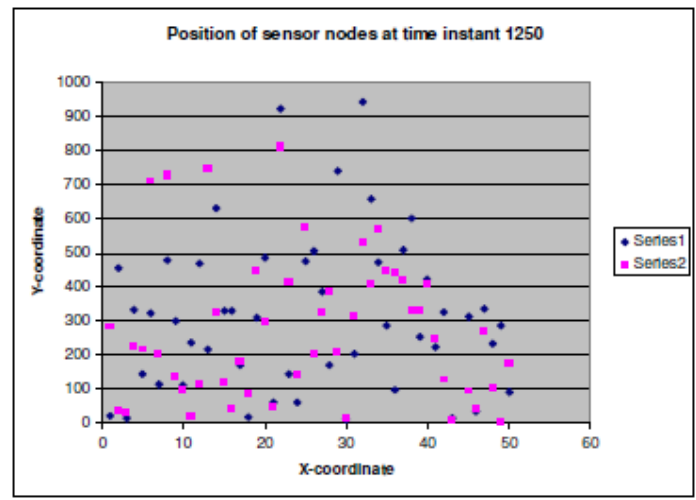

Fig. 5. Position of sensor nodes at time instance 1250 .

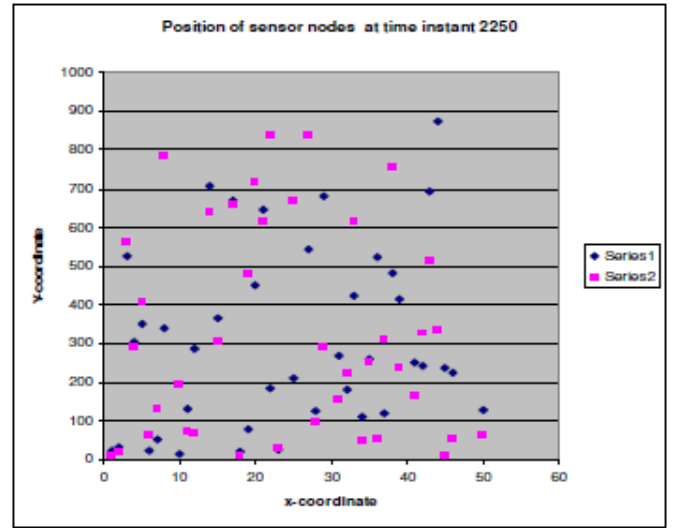

Fig. 6. Position of sensor nodes at time instance 2250 .

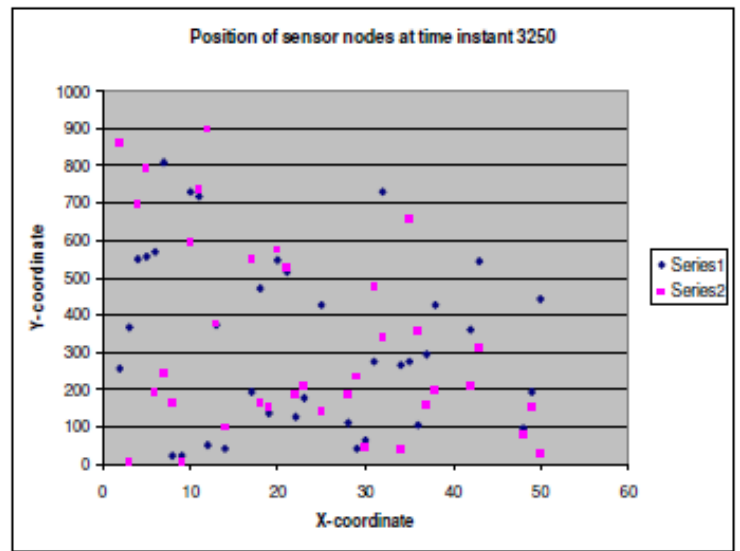

Fig. 7. Position of sensor nodes at time instance 3250.

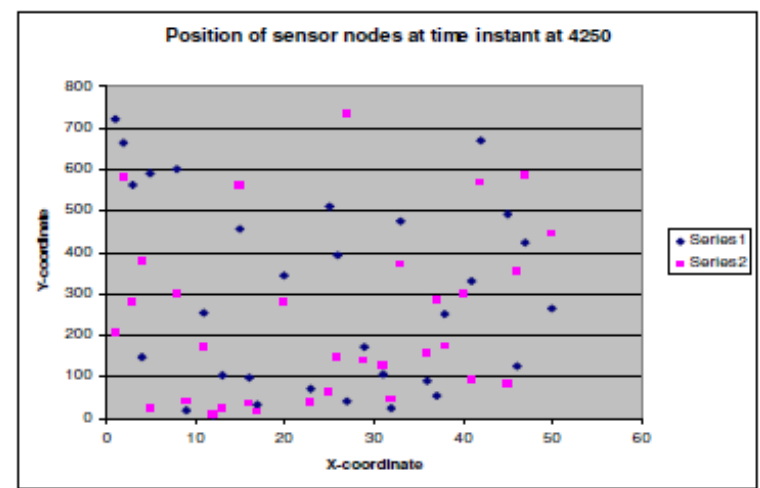

Fig. 8. Position of sensor nodes at time instance $\mathbf{4 2 5 0}$

\section{CONCLUSION}

This Mobile Sensor Networks are constituted on ad-hoc basis by tiny mobile sensor nodes deployed randomly over an area. The automatic random localization of mobile nodes may serve many potential applications where dynamicity and continuity are critically important. In this paper we envision one such application of health management. In most of the severe health risk, the patient is kept under intensive observation to collect post treatment health data about the patient. Sometime the observation period is prolonged, and just contributes to the traffic in the hospital. The housing in the central health care unit also imposes stressful cost to the patient. We propose a viable solution for the benefit of both. Post treatment under the observation period a patient - equipped with various body sensors - may be shifted to a nearby affordable locality where a patient may perform unguided longer walk also. The body sensors will continuously be disseminating the various health data to a data repository in the hospital where the concerned doctor has the access to look it up.

As the sensor networks are formed on ad-hoc basis, so they are prone for loss. A network loss causes the loss of data and absence of the health data may put the patient on severe risk. In our solution, we propose a random movement of a set of mobile sensor nodes between the body sensors and the repository and responsible to strengthen the ad-hoc wireless sensor network, the transmitting medium between them. The sensors will randomly but unobtrusively be mobile and unnoticeably strengthening the data transmission from a patient to doctor.

In this paper we attempt to find out the position of mobile sensor nodes at any instance of time. This paper solely focuses on the mobile sensor nodes. We discuss the 
advantages of the mobile sensors over the static sensors from healthcare application perspectives. Although there are few disadvantages of mobile sensors such as higher energy consumption due to sensor mobility but it is found that mobile sensors are more beneficial for the sensor network in most of the aspects. The key idea behind this paper is the scalable healthcare system on affordable cost where the deployment of large number of mobile sensors bridges the data communication from body area network to healthcare repository. If we deploy the dynamic sensor nodes in place of the static, then we are sure that with lesser number of nodes, a larger area will be covered up. If the position of each sensor is known at any point of time then the information about the patient in the health environment -affordable living facility and hospital- be updated more efficiently. In this paper we have discussed the use of mobile sensors to calculate the concentration of pollutants in a given region.

\section{REFERENCES}

[1.] Christian Bettstetter, Hannes Hartenstein, Xavier PérezCosta," Stochastic properties of the random waypoint mobility model," Proceedings of the 4th ACM international workshop on Modeling, analysis and simulation of wireless and mobile systems, 2002

[2.] Benyuan Liu, Peter Brass, Olivier Dousse, Philippe Nain, Don Towsley," Theory: Mobility improves coverage of sensor networks," Proceedings of the 6th ACM international symposium on Mobile ad hoc networking and computing MobiHoc, 2005.

[3.] B. S. Manoj, Archana Sekhar, C. Siva Ram Murthy," On the use of limited autonomous mobility for dynamic coverage maintenance in sensor networks,"Computer
Networks: International Journal of Computer Network and Telecommunications Networking, 2007.

[4.] Tai-Lin Chin, Parameswaran Ramanathan, Kewal K. Saluja," Main track: Analytic modeling of detection latency in mobile sensor networks,"Proceedings of the fifth international conference on Information processing in sensor networks, 2006.

[5.] P. Santi and D. M. Blough. "The critical transmitting range for connectivity in sparse wireless ad hoc networks." IEEE Transactions on Mobile Computing, 2003

[6.] Moshaddique Al Ameen et al., "Social Issue in Wireless Sensor Networks with Healthcare Perspective," The international Arab Journal of Information Technology, Vol.8, No.1, January 2011

[7.] Mereca Hatler et al., "WSN for Healthcare," A Market Dynamics Report, $2012 . \quad$ [Online] http://onworld.com/research/healthcare/ vip/

[8.] Sushant Jain, Rahul C. Shah, Waylon Brunette, Gaetano Borriello, Sumit Roy ," Exploiting mobility for energy efficient data collection in wireless sensor networks," Mobile Networks and Applications, 2006

[9.] Sumana Srinivasan, Krithi Ramamritham," Sensor networks: Contour estimation using collaborating mobile sensors," Proceedings of the 2006 workshop on Dependability issues in wireless ad hoc networks and sensor networks, 2006 